\title{
Is there a correlation between (18)F-FDG-PET standardized uptake value, T-classification, histological grading and the anatomic subsites in newly diagnosed squamous cell carcinoma of the head and neck?
}

\author{
Haerle, S K ; Huber, G F ; Hany, T F ; Ahmad, N ; Schmid, D T
}

\begin{abstract}
F-Fluorodeoxyglucose (FDG) positron emission tomography/computed tomography (PET)/CT imaging of squamous cell carcinoma of the head and neck (HNSCC) renders the possibility to study metabolic tumor activity by measuring FDG-uptake expressed as maximum standardized uptake value $(\mathrm{SUV}(\max ))$. A correlation between $\mathrm{SUV}(\max )$ and several factors including T-classification, histological tumor differentiation or different anatomic subsites is of potential interest in HNSCC. The aim of this study was to evaluate how metabolic tumor activity derived from FDG-PET correlates with prognostic clinical and pathological parameters including these factors. 262 patients with HNSCC undergoing $\mathrm{PET} / \mathrm{CT}$ for initial staging were assessed separately for a potential correlation between SUV $(\max )$ and Tclassification, histological grading, and anatomical subsites of the primary tumor. Nonparametric testing showed a significant correlation between $\mathrm{SUV}(\max )$ and T-classification $(\mathrm{P}<0.001)$. On the contrary, no statistically significant correlation was found between SUV( $\max )$ and histological tumor grading. Furthermore, no statistical significant correlation between the different anatomical subsites and SUV(max) were found. There was no significant correlation of SUV(max) and tumor grading after adjustment for T-stage and anatomical localization of the tumor, neither. Conclusion: Metabolic tumor activity correlates with T-stage of HNSCC. However, histological tumor grading does not correlate with SUV( $\max )$. The role of primary tumor $\mathrm{SUV}(\max )$ as a predictor of outcome or survival remains unclear. Clinicians should therefore exercise caution in attributing any clinical importance to SUV(max) obtained from a single PET/CT exam.
\end{abstract}

DOI: https://doi.org/10.1007/s00405-010-1348-2

Posted at the Zurich Open Repository and Archive, University of Zurich

ZORA URL: https://doi.org/10.5167/uzh-35583

Journal Article

Published Version

Originally published at:

Haerle, S K; Huber, G F; Hany, T F; Ahmad, N; Schmid, D T (2010). Is there a correlation between (18)FFDG-PET standardized uptake value, T-classification, histological grading and the anatomic subsites in newly diagnosed squamous cell carcinoma of the head and neck? European Archives of Oto-RhinoLaryngology, 267(10):1635-40.

DOI: https://doi.org/10.1007/s00405-010-1348-2 


\title{
Is there a correlation between ${ }^{18} \mathrm{~F}$-FDG-PET standardized uptake value, $T$-classification, histological grading and the anatomic subsites in newly diagnosed squamous cell carcinoma of the head and neck?
}

\author{
Stephan K. Haerle • G. F. Huber • T. F. Hany • \\ N. Ahmad $\cdot$ D. T. Schmid
}

Received: 20 April 2010/Accepted: 14 July 2010/Published online: 3 August 2010

(C) Springer-Verlag 2010

\begin{abstract}
F-Fluorodeoxyglucose (FDG) positron emission tomography/computed tomography (PET)/CT imaging of squamous cell carcinoma of the head and neck (HNSCC) renders the possibility to study metabolic tumor activity by measuring FDG-uptake expressed as maximum standardized uptake value $\left(\mathrm{SUV}_{\max }\right)$. A correlation between $\mathrm{SUV}_{\max }$ and several factors including T-classification, histological tumor differentiation or different anatomic subsites is of potential interest in HNSCC. The aim of this study was to evaluate how metabolic tumor activity derived from FDG-PET correlates with prognostic clinical and pathological parameters including these factors. 262 patients with HNSCC undergoing PET/CT for initial staging were assessed separately for a potential correlation between $\mathrm{SUV}_{\text {max }}$ and T-classification, histological grading, and anatomical subsites of the primary tumor. Nonparametric testing showed a significant correlation between $\mathrm{SUV}_{\text {max }}$ and T-classification $(P<0.001)$. On the contrary, no statistically significant correlation was found between $\mathrm{SUV}_{\text {max }}$ and histological tumor grading. Furthermore, no statistical significant correlation between the different anatomical subsites and $\mathrm{SUV}_{\max }$ were found. There was no significant correlation of $\mathrm{SUV}_{\max }$ and tumor grading after adjustment for T-stage and anatomical localization of the tumor, neither. Conclusion: Metabolic tumor activity
\end{abstract}

S. K. Haerle $(\bowtie)$ · G. F. Huber · N. Ahmad

Department of Otolaryngology, Head and Neck Surgery,

University Hospital Zurich, Frauenklinikstrasse 24,

8091 Zurich, Switzerland

e-mail: Stephan.Haerle@usz.ch

T. F. Hany · D. T. Schmid

Division of Nuclear Medicine, Department of Medical

Radiology, University Hospital Zurich, Raemistrasse 100,

8091 Zurich, Switzerland correlates with T-stage of HNSCC. However, histological tumor grading does not correlate with $\mathrm{SUV}_{\max }$. The role of primary tumor $\mathrm{SUV}_{\max }$ as a predictor of outcome or survival remains unclear. Clinicians should therefore exercise caution in attributing any clinical importance to $\mathrm{SUV}_{\text {max }}$ obtained from a single PET/CT exam.

Keywords FDG-PET/CT •

Standardized uptake value (SUV) .

Squamous cell carcinoma $\cdot$ Head and neck

\section{Introduction}

Many different staging methods for squamous cell carcinoma of the head and neck (HNSCC) have been described over the last two decades. Clinical staging includes panendoscopy and different imaging modalities, such as computed tomography (CT) or magnetic resonance imaging (MRI), both available at most institutions. Combined functional and morphological imaging, such as ${ }^{18}$ F-Fluorodeoxyglucose (FDG) positron emission tomography (PET)/CT for staging of HNSCC has shown promising results recently [1-4]. In addition to conventional morphological imaging using CT and MRI, PET scanning allows for non-invasive study of tumor physiology [5]. There is a correlation between the uptake of FDG and the level of intracellular metabolic tumor activity, which in turn may be associated with active proliferation, invasion and incidence of loco-regional as well as distant metastases [6]. To quantify the amount of FDG uptake within a region of interest, the standardized uptake value (SUV) is used most commonly, since the measurement is standardized for injected activity, body weight and decay time. Corresponding, correlation between $\mathrm{SUV}_{\max }$ and 
several factors including T-classification, histological tumor differentiation or different anatomic subsites is of potential interest in HNSCC. Early detection of HNSCC provides a significant survival advantage and the site of primary disease is an important determinant of survival for most cancers of the head and neck [7]. Recent studies looked at different prognostic factors, whereas one of the outmost important prognostic factors for surviving HNSCC is the cervical lymph node involvement. Interestingly, Chen et al. [8] reported a higher prevalence of nodal metastases in elective neck dissections for poorly differentiated carcinomas when compared to well-differentiated tumors. Additionally, in a recent study Goerkem et al. [9] looked at the rate of occult metastases in the context of sentinel node biopsy: the less differentiated the primary was, the higher was the risk for developing occult metastases. Newer studies have included the maximum SUV ( $\left(\mathrm{SUV}_{\max }\right)$ in the context for predicting outcome for patient suffering from HNSCC by looking at early SUV pattern changes after multimodality treatment $[6,10]$. In their study, Suzuki et al. [10] also described a correlation between the $\mathrm{SUV}_{\max }$ and T-stage.

The aim of this study was to evaluate how metabolic tumor activity $\left(\mathrm{SUV}_{\max }\right)$ derived from FDG-PET correlates with prognostic clinical and pathological parameters including T-classification, histological tumor differentiation or different anatomic subsites in HNSCC at the time point of initial staging. This is important to understand if metabolic information obtained from a single PET/CT exam has the potential to serve as a predictor of disease outcome and possibly influence initial treatment strategy or if it only helps to differentiate benign from malignant changes.

\section{Patients and methods}

Two head and neck surgeons (HSK, HGF) reviewed the charts of all patients presenting for initial treatment of a HNSCC between November 2001 and December 2007, retrospectively. A board-certified physician in both nuclear medicine and radiology (SDT) reviewed records of all FDG-PET/CT exams. A total of 294 patients with histological confirmed and previously untreated HNSCC underwent FDG-PET/CT for initial staging. All patients in our study granted permission for anonymized evaluation of their medical data for scientific purposes prior to PET/CT imaging.

This patient cohort does not represent a consecutive group of all patients diagnosed with HNSCC within the given time frame, because mainly patients with advanced disease (T3/T4 and N2/N3, respectively) were referred for FDG-PET/CT to search for loco-regional lymph node metastases, distant metastases and synchronous malignant tumors.
Imaging protocol

A combined PET/CT inline system (Discovery LS or Discovery ST, GE Healthcare) was used for this study. This device integrates a PET scanner (GE Advance Nxi; GE Healthcare) with a multi-slice helical CT (LightSpeed Plus or LightSpeed 16; GE Healthcare) and permits the acquisition of co-registered CT and PET images in the same session.

Patients fasted for at least $4 \mathrm{~h}$ prior to scanning, which started $60 \mathrm{~min}$ after the injection of a standard dose of approximately $350 \mathrm{MBq}$ of FDG. An oral CT contrast agent was given $75-60 \mathrm{~min}$ before scanning $(150 \mathrm{~mL}$, Micropaque, Guerbet; diluted with $850 \mathrm{~mL}$ water). All patients were examined in the supine position with arms down. The CT scan was acquired during breath holding or free shallow breathing. In the normal expiratory position, the following parameters were used $140 \mathrm{kV}, 80 \mathrm{~mA}, 0.5 \mathrm{~s}$ tube rotation, $4.25-\mathrm{mm}$ section thickness, a scan length of $867 \mathrm{~mm}$ with $22.5 \mathrm{~s}$ scan time. Acquisition of the FDGPET scan was started immediately after the CT with an emission time of $3 \mathrm{~min}$ per table position resulting in a total scan time of $18 \mathrm{~min}$ for 6 table positions from the head to the pelvic floor.

CT data was used for attenuation correction. PET images were reconstructed using a standard 2-dimensional iterative algorithm [ordered subset expectation maximization (OSEM)].

\section{$\mathrm{SUV}_{\max }$ analysis}

A board-certified physician in both nuclear medicine and radiology with 6 years experience in $\mathrm{CT}$ reading and 3 years experience in reading combined FDG-PET/CT in head and neck cancer patients determined the $\mathrm{SUV}_{\text {max }}$ retrospectively. Calculation of $\mathrm{SUV}_{\max }$ was done using a commercially available workstation (Advantage workstation, software version 4.4, GE Healthcare). Before measurement, a head and neck surgeon determined the correct localization of the primary tumor retrospectively based on the knowledge of intraoperative/invasive procedures performed after the PET/CT scan. A volume of interest (VOI) was defined on screen that included the FDG uptake related to the primary tumor on multiple consecutive image sections. The value of the single voxel with the highest activity within this VOI was used for calculation of $\mathrm{SUV}_{\max }$ (Fig. 1). The software provides this value automatically. All values were normalized to the lean body mass (LBM) of the patient. The use of LBW instead of body weight has been shown to be less dependent on the body habitus of the study population [11]. LBM was determined using a personal scale with an integrated foot-to-foot bioelectric 

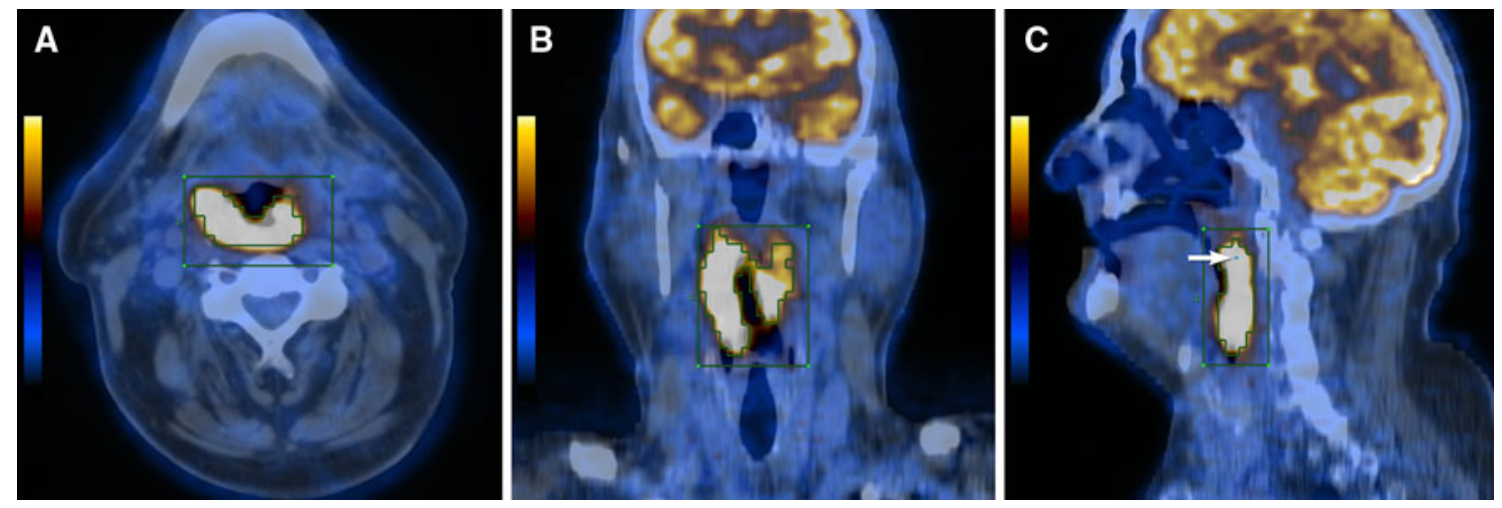

Fig. 1 Axial (a), coronal (b) and sagittal (c) fused FDG-PET/CT images of a 71-year old male patient with a newly diagnosed oropharyngeal carcinoma illustrating the measurement of SUVmax: the volume of interest (green rectangles) was set to enclose the whole

impedance analyzer (Tanita model 2001; Tanita, Tokyo, Japan).

\section{Histological grading}

The histological work-up of the primary was performed according to the guidelines of the Swiss Society of Pathology. All specimens were sectioned along the greatest diameter and paraffin-embedded. Whole sections were stained with hematoxylin-eosin, thus two surfaces per biopsy were available. The specimens were subdivided into well differentiated (1), moderately differentiated (2), and poorly differentiated (3).

\section{Statistical analysis}

The entire cohort was analyzed according to the following endpoints: the correlation between $\mathrm{SUV}_{\max }$ and T-stage and the potential correlation between $\mathrm{SUV}_{\max }$ and the histological grading with and without adjustment for anatomical localization and T-stage. Furthermore, we have analyzed the correlation between $\mathrm{SUV}_{\max }$ and the anatomical subtype of the primary. Nonparametric testing was used. $P$ values $<0.05$ were considered significant. All calculations were carried out using SPSS version 17.0 for windows.

\section{Results}

\section{Clinical parameters}

Between 2001 and 2007, a total number of 294 patients were evaluated with FDG-PET/CT for a previously untreated HNSCC. 17 patients were excluded due to missing data for SUV calculation (lean body weight, patient's size, or injected activity), 2 patients were tumor. A threshold of $25 \%$ of the SUVmax was chosen to outline the metabolic active tumor volume (green line within the green rectangle). The software automatically determined the hottest voxel within this volume (c, white arrow)

excluded because of missing PET data, 1 patient was excluded because of CUP (cancer of unknown primary) syndrome, and 12 patients were excluded because the primary tumor was not FDG avid in PET imaging (11 patients suffering from a $\mathrm{T} 1$ lesion, 1 patient suffering from a T2 lesion). Finally, the number of 262 patients remained for evaluation. There was a male predominance $(n=204$, $78 \%$ ). The mean age was 60.0 years (standard deviation \pm 10 years, range $36-90$ years).

\section{Localization and extension of the primary}

In both male and female patients, the primary tumor was predominantly located in the oropharynx $(n=144,55 \%)$, followed by the hypopharynx $(n=51,19 \%)$, the larynx $(n=33,13 \%)$, the oral cavity $(n=30,11 \%)$, and the nasopharynx $(n=4,2 \%)$. All patients presented in the advanced stages III and IV [International Union against Cancer (UICC) 1997]: $n=41(16 \%)$ patients were staged as stage III, $n=189(72 \%)$ patients were staged as stage IVa, and $n=32(12 \%)$ patients were staged as stage IVb. $\mathrm{T}$ - and N-stages of all tumors are shown in Table 1.

\section{Histological differentiation of the primary tumors}

Out of the 262 primary tumors, $15(6 \%)$ were well-differentiated SCC, 141 (54\%) were moderately differentiated, and $106(40 \%)$ of all primary tumors were found to be poorly differentiated.

Mean $\mathrm{SUV}_{\max }$ values

$\mathrm{SUV}_{\max }$ of all primaries was 10.7 (standard deviation \pm 4.6 , range 2.9-33.7). Mean $\mathrm{SUV}_{\max }$ for the different anatomic subsites, the different T-stages and histological gradings are listed in Table 2 . 
Table 1 T- and N-stages of all patients $(n=262)$ based on all available clinical examination including panendoscopy, contrast-enhanced CT and PET/ CT

Data are numbers of subjects, with percentages in parentheses

\begin{tabular}{lcrlllrr}
\hline & $\mathrm{N} 0$ & $\mathrm{~N} 1$ & $\mathrm{~N} 2 \mathrm{a}$ & $\mathrm{N} 2 \mathrm{~b}$ & \multicolumn{1}{l}{$\mathrm{N} 2 \mathrm{c}$} & \multicolumn{1}{c}{$\mathrm{N} 3$} & \multicolumn{1}{c}{ Total } \\
\hline $\mathrm{T} 1$ & $0(0)$ & $8(3.1)$ & $3(1.1)$ & $16(6.1)$ & $2(0.8)$ & $2(0.8)$ & $31(11.8)$ \\
$\mathrm{T} 2$ & $0(0)$ & $10(3.8)$ & $0(0)$ & $66(25.2)$ & $12(4.6)$ & $5(1.9)$ & $93(35.5)$ \\
$\mathrm{T} 3$ & $15(5.7)$ & $8(3.1)$ & $1(0.4)$ & $25(9.5)$ & $14(5.3)$ & $1(0.4)$ & $64(24.4)$ \\
$\mathrm{T} 4$ & $11(4.2)$ & $10(3.8)$ & $2(0.8)$ & $23(8.8)$ & $23(8.8)$ & $5(1.9)$ & $74(28.2)$ \\
Total & $26(9.9)$ & $36(13.7)$ & $6(2.3)$ & $130(49.6)$ & $51(19.5)$ & $13(5.0)$ & $262(100)$ \\
\hline
\end{tabular}

Table 2 Clinical characteristics and correlations between $\mathrm{SUV}_{\max }$ and T-classification, histological grading, and the anatomic subsite a Data are numbers of subjects, with percentages in parentheses

${ }^{b}$ Data are means $\pm \mathrm{SD}$, with range in parentheses

c Kruskal-Wallis test

\begin{tabular}{lccc}
\hline Characteristics & Patients $^{\mathrm{a}}$ & SUV $_{\max }^{\mathrm{b}}$ & $P$ value \\
\hline T-stage & & & \\
T1 & $31(11.8)$ & $8.3 \pm 3.2(3.0-21.8)$ & $<0.001^{\mathrm{c}}$ \\
T2 & $93(35.5)$ & $10.2 \pm 4.5(2.9-24.7)$ & \\
T3 & $64(24.4)$ & $11.0 \pm 4.3(4.0-23.6)$ & \\
T4 & $74(28.2)$ & $11.9 \pm 4.8(3.4-33.7)$ & \\
Histological grading & & & \\
Well differentiated & $15(5.7)$ & $12.0 \pm 4.1(3.8-16.4)$ & \\
Moderately differentiated & $141(53.8)$ & $10.4 \pm 4.4(2.9-33.7)$ & \\
Poorly differentiated & $106(40.5)$ & $10.9 \pm 5.0(3.7-29.9)$ & \\
Anatomical subsite & & & \\
Nasopharynx & $4(1.5)$ & $11.5 \pm 2.2(8.3-13.3)$ & \\
Oral cavity & $30(11.5)$ & $11.4 \pm 4.4(3.4-23.6)$ & \\
Oropharynx & $144(55.0)$ & $10.4 \pm 4.5(2.9-33.7)$ & \\
Hypopharynx & $51(19.5)$ & $10.7 \pm 5.0(3.8-24.7)$ & \\
Larynx & $33(12.6)$ & $11.4 \pm 5.1(4.1-29.9)$ & \\
\hline
\end{tabular}

Correlation between $\mathrm{SUV}_{\max }$ and endpoints

Nonparametric testing using Kruskal-Wallis test showed a significant correlation $(P<0.001)$ of $\mathrm{SUV}_{\max }$ and T-stage. There was no statistically significant correlation of $\mathrm{SUV}_{\max }$ and histological grading $(P=0.232)$. Furthermore, no significant differences were found for mean $\mathrm{SUV}_{\max }$ of all tumors of the same stage in relation to the anatomic subsite of the primary tumor with $P=0.133$ for T1 tumors $(n=31), P=0.626$ for T2 tumors $(n=93), P=0.578$ for T3 tumors $(n=64)$ and $P=0.721$ for T4 tumors $(n=74)$. There was no significant correlation between the different anatomic subsites and mean $\operatorname{SUV}_{\text {max }}(P=0.646)$. Furthermore, there was no significant correlation of $\mathrm{SUV}_{\max }$ and tumor grading after adjustment for T-stage and anatomical localization of the tumor.

\section{Discussion}

FDG-PET/CT marks an essential tool for initial staging in advanced HNSCC. Many important questions, such as the presence of second primaries and distant metastases can be answered at the time of diagnosis by using FDG-PET/CT
[2]. Therefore, FDG-PET/CT has a direct impact on therapy decision and outcome [12]. Within the last few years, the literature shows a growing tendency to take into account metabolic information about the tumor when planning treatment of HNSCC and predicting patient's outcome [13].

The aim of this study was to assess a correlation between different clinical and pathological parameters and $\mathrm{SUV}_{\max }$. This may play an important role regarding the latest theories suggesting that FDG uptake of the primary tumor is related to the treatment response, and survival in HNSCC [14-17]. $\mathrm{SUV}_{\max }$ is the most widely used parameter to measure metabolic tumor activity in oncologic FDG-PET/CT imaging. According to Machtay et al. [6] low $\mathrm{SUV}_{\max }$ of the primary tumor was significantly associated with longer disease-free survival after radiotherapy of HNSCC in a series of 60 patients. On the contrary, Suzuki et al. [10] found no correlation between SUV $_{\text {max }}$ and treatment outcome in HNSCC after radiotherapy in a series of 45 patients. Therefore, to elucidate the role of $\mathrm{SUV}_{\max }$ and its possible predictive role for therapy response more distinguishable, the aim of this retrospective analysis was to assess a potential correlation between $\mathrm{SUV}_{\max }$ and different clinical and pathological 


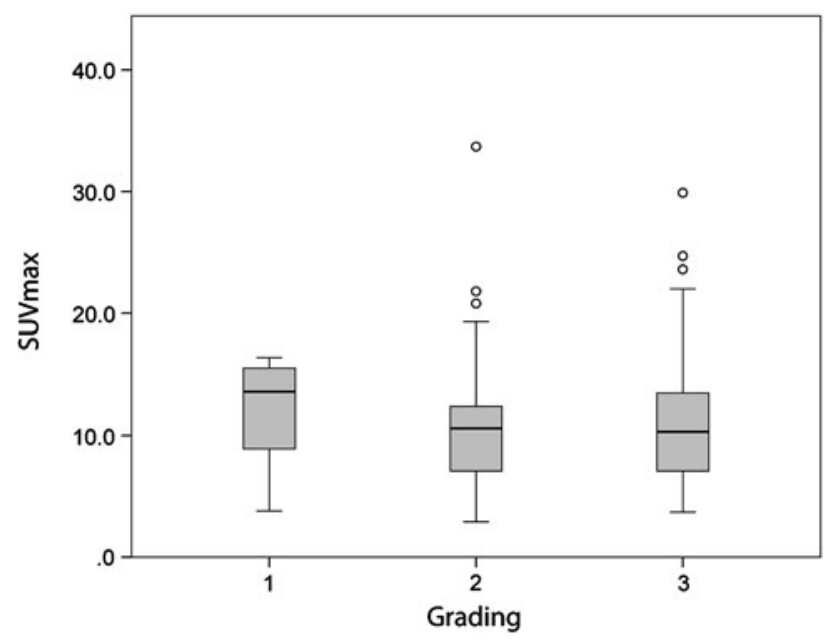

Fig. 2 Box-Plot illustrating the relation of $S U V_{\max }$ and histological grading of the primary tumors $(n=262) .15(6 \%)$ tumors were well differentiated (grade 1), 141 (54\%) moderately differentiated (grade 2 ) and $106(40 \%)$ were poorly differentiated (grade 3)

parameters at initial staging in a large patient cohort. First, a positive correlation between $\mathrm{SUV}_{\max }$ and T-stage can be shown $(P<0.001)$. In other words: the higher the T-stage, the higher the $\mathrm{SUV}_{\max }$. This is in accordance with other authors $[10,15,18,19]$. A recent study in 627 patients with oropharyngeal carcinoma identified higher T-stage as a prognostic factor for poorer local control [20]. With regard to the histological grading of HNSCC and $\mathrm{SUV}_{\max }$ no statistical correlation was found in our series $(P=0.232)$. This is in accordance to the findings of Suzuki et al. [10]. However, $\mathrm{SUV}_{\max }$ values greater than 16.4 were only observed in 24 moderately and poorly differentiated tumors in our series of patients but not in well differentiated tumors (Fig. 2). The idea to look at this potential correlation lies in the fact of the helpful discriminating ability of FDG-PET/CT in the evaluation of grading of soft-tissue sarcoma [21]. Furthermore, survival is improved in patients with differentiated tumors as compared to those with poorly differentiated tumors [22]. However, summarizing our results within a relatively large patient cohort $(n=262)$, we did not find any correlation between histopathological aggressiveness and $\mathrm{SUV}_{\max }$. Nevertheless, there are authors proposing a cutoff SUV value of 9 correlating with worse survival [19]. The last parameter we looked at was the anatomic subsite of HNSCC. Again, no significant correlation between $\mathrm{SUV}_{\max }$ and tumor subsite was found $(P=0.646)$. To us, this is not surprising since a T3 tonsillar carcinoma with avid FDG uptake is not comparable to a T3 carcinoma of the larynx where FDG uptake may not be as high due to the different definitions of a T3 tumor at different subsites. Further studies are needed to assess the possible value of $\mathrm{SUV}_{\max }$ as a predictive factor of treatment outcome in HNSCC.

\section{Conclusion}

Metabolic tumor activity measured with FDG-PET/CT and expressed as $\mathrm{SUV}_{\max }$ correlates with T-stage of HNSCC. However, histological tumor grading does not correlate with $\mathrm{SUV}_{\max }$. The role of primary tumor $\mathrm{SUV}_{\max }$ as a predictor of outcome or survival remains unclear. Clinicians should therefore exercise caution in attributing any clinical importance to $\mathrm{SUV}_{\max }$ obtained from a single PET/ CT exam.

Conflict of interest The authors declare that they have no conflict of interest.

\section{References}

1. Strobel K, Haerle SK, Stoeckli SJ, Schrank M, Soyka JD, VeitHaibach P, Hany TF (2009) Head and neck squamous cell carcinoma (HNSCC): detection of synchronous primaries with (18)F-FDG-PET/CT. Eur J Nucl Med Mol Imaging 36:919-927. doi:10.1007/s00259-009-1064-6

2. Haerle SK, Strobel K, Hany TF, Sidler D, Stoeckli SJ (2010) (18)F-FDG-PET/CT versus panendoscopy for the detection of synchronous second primary tumors in patients with head and neck squamous cell carcinoma. Head Neck 32:319-325. doi:10.1002/hed.21184

3. Lonneux M, Hamoir M, Reychler H, Maingon P, Duvillard C, Calais G, Bridji B, Digue L, Toubeau M, Gregoire V (2010) Positron emission tomography with [18F]fluorodeoxyglucose improves staging and patient management in patients with head and neck squamous cell carcinoma: a multicenter prospective study. J Clin Oncol 28:1190-1195. doi:10.1200/JCO.2009.24. 6298

4. Uyl-de Groot CA, Senft A, de Bree R, Leemans CR, Hoekstra OS (2010) Chest CT and whole-body 18F-FDG PET are cost-effective in screening for distant metastases in head and neck cancer patients. J Nucl Med 51:176-182. doi:10.2967/jnumed.109. 067371

5. Rohren EM, Turkington TG, Coleman RE (2004) Clinical applications of PET in oncology. Radiology 231:305-332. doi:10.1148/radiol.2312021185

6. Machtay M, Natwa M, Andrel J, Hyslop T, Anne PR, Lavarino J, Intenzo CM, Keane W (2009) Pretreatment FDG-PET standardized uptake value as a prognostic factor for outcome in head and neck cancer. Head Neck 31:195-201. doi:10.1002/hed.20942

7. Maccomb W, Fletcher G (196) Cancer of the head and neck. Williams \& Wilkins Company, Baltimore, pp 152-292

8. Chen RB, Suzuki K, Nomura T, Nakajima T (1993) Flow cytometric analysis of squamous cell carcinomas of the oral cavity in relation to lymph node metastasis. J Oral Maxillofac Surg 51:397-401

9. Goerkem M, Braun J, Stoeckli SJ (2010) Evaluation of clinical and histomorphological parameters as potential predictors of occult metastases in sentinel lymph nodes of early squamous cell carcinoma of the oral cavity. Ann Surg Oncol 17:527-535. doi:10.1245/s10434-009-0755-3

10. Suzuki K, Nishioka T, Homma A, Tsuchiya K, Yasuda M, Aoyama H, Onimaru R, Tamaki N, Shirato H (2009) Value of fluorodeoxyglucose positron emission tomography before radiotherapy for head and neck cancer: does the standardized uptake value predict treatment outcome? Jpn J Radiol 27:237-242. doi:10.1007/ s11604-009-0330-7 
11. Sugawara Y, Zasadny KR, Neuhoff AW, Wahl RL (1999) Reevaluation of the standardized uptake value for FDG: variations with body weight and methods for correction. Radiology 213:521-525

12. Schmid DT, Stoeckli SJ, Bandhauer F, Huguenin P, Schmid S, von Schulthess GK, Goerres GW (2003) Impact of positron emission tomography on the initial staging and therapy in locoregional advanced squamous cell carcinoma of the head and neck. Laryngoscope 113:888-891. doi:10.1097/00005537-20030500000021

13. Hustinx R, Lucignani G (2010) PET/CT in head and neck cancer: an update. Eur J Nucl Med Mol Imaging 37:645-651

14. Kitagawa Y, Sano K, Nishizawa S, Nakamura M, Ogasawara T, Sadato N, Yonekura Y (2003) FDG-PET for prediction of tumour aggressiveness and response to intra-arterial chemotherapy and radiotherapy in head and neck cancer. Eur J Nucl Med Mol Imaging 30:63-71. doi:10.1007/s00259-002-0978-z

15. Halfpenny W, Hain SF, Biassoni L, Maisey MN, Sherman JA, McGurk M (2002) FDG-PET. A possible prognostic factor in head and neck cancer. Br J Cancer 86:512-516. doi:10.1038/ sj.bjc. 6600114

16. Brun E, Kjellen E, Tennvall J, Ohlsson T, Sandell A, Perfekt R, Wennerberg J, Strand SE (2002) FDG PET studies during treatment: prediction of therapy outcome in head and neck squamous cell carcinoma. Head Neck 24:127-135. doi:10.1002/ hed. 10037
17. Allal AS, Dulguerov P, Allaoua M, Haenggeli CA, El-Ghazi el A, Lehmann W, Slosman DO (2002) Standardized uptake value of 2 -[(18)F] fluoro-2-deoxy-D-glucose in predicting outcome in head and neck carcinomas treated by radiotherapy with or without chemotherapy. J Clin Oncol 20:1398-1404

18. Torizuka T, Tanizaki Y, Kanno T, Futatsubashi M, Naitou K, Ueda Y, Ouchi Y (2009) Prognostic value of 18F-FDG PET in patients with head and neck squamous cell cancer. AJR Am J Roentgenol 192:W156-W160. doi:10.2214/AJR.08.1429

19. Schwartz DL, Rajendran J, Yueh B, Coltrera MD, Leblanc M, Eary J, Krohn K (2004) FDG-PET prediction of head and neck squamous cell cancer outcomes. Arch Otolaryngol Head Neck Surg 130:1361-1367. doi:10.1001/archotol.130.12.1361

20. Agarwal JP, Mallick I, Bhutani R, Ghosh-Laskar S, Gupta T, Budrukkar A, Murthy V, Sengar M, Dinshaw KA (2009) Prognostic factors in oropharyngeal cancer-analysis of 627 cases receiving definitive radiotherapy. Acta Oncol 48:1026-1033. doi: $10.1080 / 02841860902845839$

21. Ioannidis JP, Lau J (2003) 18F-FDG PET for the diagnosis and grading of soft-tissue sarcoma: a meta-analysis. J Nucl Med 44:717-724

22. Ensley J, Crissman J, Kish J, Jacobs J, Weaver A, Kinzie J, Cummings G, Al-Sarraf M (1986) The impact of conventional morphologic analysis on response rates and survival in patients with advanced head and neck cancers treated initially with cisplatin-containing combination chemotherapy. Cancer 57:711-717 